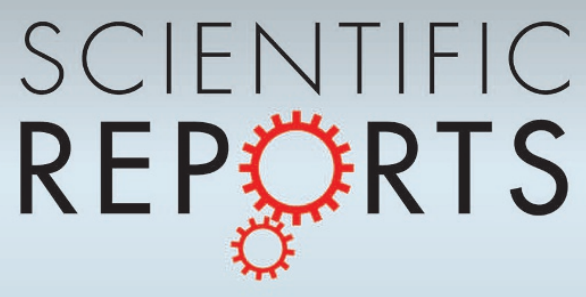

\title{
OPEN A microfibre assembly of an iron-carbon composite with giant magnetisation
}

SUBJECT AREAS:

SENSORS AND

BIOSENSORS

APPLIED PHYSICS

ELECTRICAL AND ELECTRONIC

ENGINEERING

SYNTHESIS AND PROCESSING

Received

20 June 2013

Accepted

10 October 2013

Published

29 October 2013

Correspondence and requests for materials should be addressed to G.W.Y. (stsygw@mail. sysu.edu.cn)

* These authors contributed equally to this work.

\author{
Ying Liang*, Pu Liu*, Jun Xiao, Hongbo Li, Chengxin Wang \& Guowei Yang
}

State Key Laboratory of Optoelectronic Materials and Technologies, Institute of Optoelectronic and Functional Composite Materials, Nanotechnology Research Center, School of Physics \& Engineering, Sun Yat-sen University, Guangzhou 51 0275 , Guangdong, P. R. China.

Iron carbide is among the oldest known materials. The utility of this ancient advanced material is greatly extended in its nanostructured forms. We demonstrate for the first time that one-dimensional iron carbide microfibres can be assembled in liquid using strong magnetic field-assisted laser ablation. The giant saturation magnetisation of these particles was measured a $261 \mathrm{emu} / \mathrm{g}$ at room temperature, which is the best value reported to date for iron nitride and carbide nanostructures, is 5.5 times greater than the 47 $\mathrm{emu} / \mathrm{g}$ reported for $\mathrm{Fe}_{3} \mathrm{C}$ nanoparticles, and exceeds the $212 \mathrm{emu} / \mathrm{g}$ for bulk Fe. The magnetic field-induced dipolar interactions of the magnetic nanospheres and the nanochains played a key role in determining the shape of the product. These findings lead to a variety of promising applications for this unique nanostructure including its use as a magnetically guided transporter for biomedicine and as a magnetic recording material.

nterest in the self-assembly of magnetic nanoparticles (MNPs) into larger ordered structures as an approach for engineering new materials and devices ${ }^{1}$ such as photonic crystals ${ }^{2}$, magnetotransporters ${ }^{3}$, micromechanical sensors ${ }^{4}$, magnetic memory materials ${ }^{5}$, magnetic switching devices ${ }^{6}$, magnetic force probes ${ }^{7}$, and DNA separation materials ${ }^{8}$ among others ${ }^{9,10}$ has recently increased. Therefore, developing generally applicable and effective techniques for the assembly of ordered nanostructures of MNPs is essential. For nanochain or chain-like MNP nanostructures, achieving an anisotropic one-dimensional (1D) assembly from isotropic, zero-dimensional MNPs remains challenging ${ }^{11,12}$. Therefore, an effective and repeatable assembly of $1 \mathrm{D}$ MNP chains is a significant step toward realising their potential as new materials in practical applications. A number of versatile routes have been established to produce a variety of 1D MNP chains using either direct interactions (e.g., interparticle forces) or indirect methods requiring a template or an external field ${ }^{12-16}$. The use of external magnetic fields to induce the MNP assembly is simple, clean, and inexpensive ${ }^{17}$. We developed a unique and facile approach for assembling the 1D MNP chains using magnetic field-assisted laser ablation in liquid (MF-LAL). Using this technique, we assembled micro-fibres of submicron iron carbide spheres and characterised their magnetic properties. The assembled microfibres were ferromagnetic, with giant magnetisations of 261 and $295 \mathrm{emu} / \mathrm{g}$ at $300 \mathrm{~K}$ and $5 \mathrm{~K}$, respectively, which are the best values reported to date for iron nitride and carbide nanostructures ${ }^{18}$. Additionally, MF-LAL is simple, green, and catalyst-free allowing researchers to choose and design interesting solid targets and solution environments to fabricate ordered magnetic nanostructures for fundamental research and potential applications.

\section{Results}

Structure and morphology of the as-assembled microfibres. This study systematically examines the influence of the important fabrication parameters including the magnetic field, the repeating frequency, and the pulse energy of the pulsed-laser. Typical microfibres were fabricated under various conditions. Figure la-b demonstrates the influence of the magnetic field intensity on the fabrication when all other experimental conditions are held constant. Under a reduced magnetic field (1 T in Fig. 1a), the microfibres are short and bent compared with those under a higher magnetic field (5 T in Fig. 1b), which indicates that a strong magnetic field favours the fabrication of long, straight microfibres. Similarly, Figure 1c-d demonstrates the influence of the repeating laser frequency on the fabrication of the microfibres. We observed no significant difference between $2 \mathrm{~Hz}$ (Fig. 1c) and $10 \mathrm{~Hz}$ (Fig. 1d). However, the laser ablation in ethanol at the higher repeating frequency results in the undesirable burning of ethanol. A low repeating frequency reduces the product yield and further affects the formation of the microfibres. Thus, we hypothesise that a median repeating frequency is preferable. Moreover, a low energy is likely desirable for the formation of small and uniform-sized nanoparticles as shown in Fig. 1e (50 mJ) and $\mathrm{f}$ 


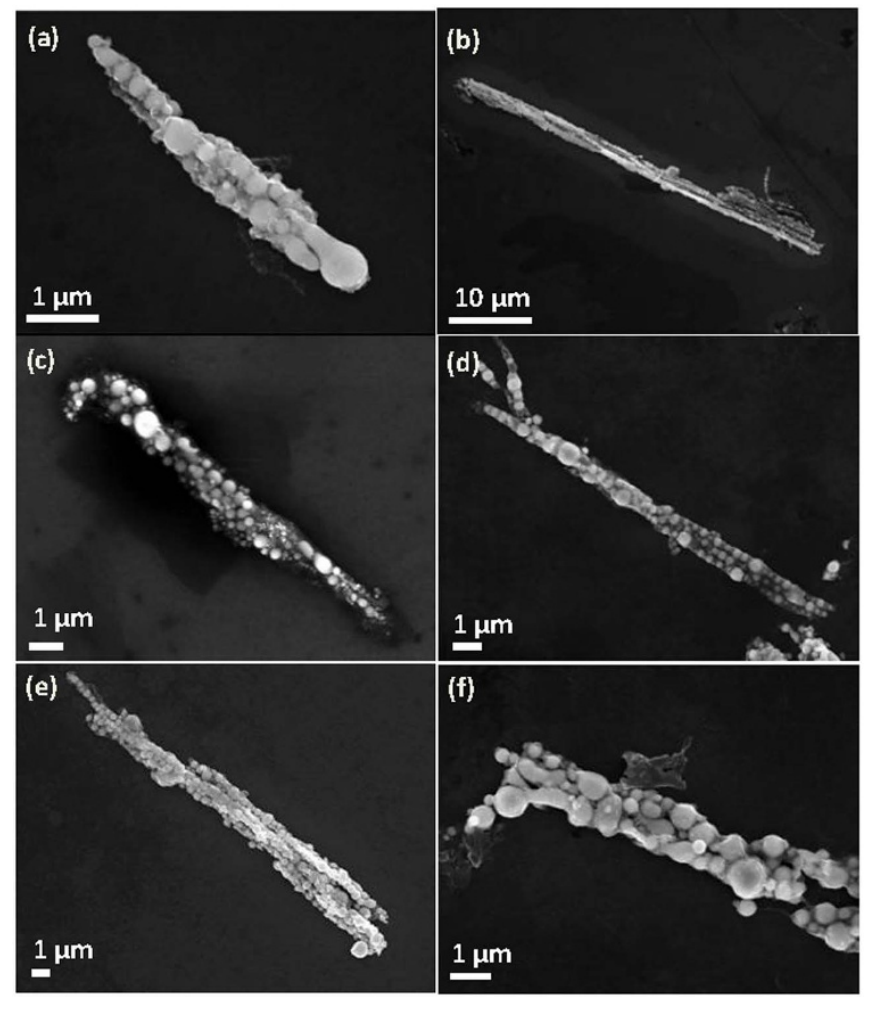

Figure $1 \mid$ SEM images of the micro-fibers under different experimental conditions. (a) $1 \mathrm{~T}, 100 \mathrm{~mJ}$ and $5 \mathrm{~Hz}$; (b) $5 \mathrm{~T}, 100 \mathrm{~mJ}$ and $5 \mathrm{~Hz}$; (c) $2 \mathrm{~Hz}$, $9 \mathrm{~T}$ and $100 \mathrm{~mJ}$; (d) $10 \mathrm{~Hz}, 9 \mathrm{~T}$ and $100 \mathrm{~mJ}$; (e) $50 \mathrm{~mJ}, 9 \mathrm{~T}$ and $5 \mathrm{~Hz}$; (f) $150 \mathrm{~mJ}, 9 \mathrm{~T}$ and $5 \mathrm{~Hz}$.

$(150 \mathrm{~mJ})$. However, the size of the nanoparticles can also be largely affected by the laser irradiation during the ablation process. Based on our systemic analysis, we can conclude that that a magnetic field of $9 \mathrm{~T}$, a pulse repeating frequency of $5 \mathrm{~Hz}$, and a pulse repeating energy of $100 \mathrm{~mJ}$ are the optimal experimental parameters for assembling the microfibre chain via MF-LAL. The products assembled under these conditions are presented in Fig. 2a-e and were several tens of micrometres long $(\sim 20-60 \mu \mathrm{m})$ and several micrometres in diameter. These ordered microfibres were constructed from 1D chains of spheres tightly bundled in an orderly fashion as depicted in Fig. 2b. We thus deduced that these microstructures consisted of linear, bunched chains of interconnected spherical particles. Spherical nanoparticles are often produced using $\mathrm{LAL}^{19}$. The average size of the as-synthesised spheres was approximately $200-300 \mathrm{~nm}$, as shown in Fig. 2c. Careful observations revealed some nanospheres with diameters of $\sim 10-20 \mathrm{~nm}$ on the surfaces of the nanochains. Some of the nanospheres were stretched along the chain direction induced by the magnetic field. The corresponding energy-dispersive X-ray spectrometer (EDS) pattern (Fig. 2d) indicated that the microfibres were primarily composed of $\mathrm{Fe}, \mathrm{C}, \mathrm{O}$, and $\mathrm{Si}$. The $\mathrm{Si}$ originated from the silicon substrate upon which the sample was deposited, whereas the $\mathrm{O}$ resulted from the small amount of oxidation that occurred. The crystalline structure of the products was characterised using X-ray powder diffraction (XRD), as shown in Fig. 1e; the products exhibited good crystallinity and were composed of the $\mathrm{Fe}_{3} \mathrm{C}$ (JCPDS No. 35-772) and $\mathrm{Fe}_{5} \mathrm{C}_{2}$ (JCPDS No. 36-1248) iron carbides. The $\mathrm{XRD}$ results suggested that the $\mathrm{Fe}_{3} \mathrm{C}$ iron carbide was predominant in the products. We thus concluded that the products consisted of $1 \mathrm{D}$ microfibres of $\mathrm{Fe}_{3} \mathrm{C}$ and $\mathrm{Fe}_{5} \mathrm{C}_{2}$ submicron spheres.

The detailed structure of the microfibres was further revealed by the images shown in Fig. 3a-f, in which a single fibre exhibits both visible interparticle spacing between adjacent spheres $(\sim 10-30 \mathrm{~nm})$ and the highly directed formation of a regular chain structure. The corresponding EDS pattern in Fig. 2b displays the elements $\mathrm{Fe}, \mathrm{C}$, and $\mathrm{Cu}$. The $\mathrm{Cu}$ originated from the copper mesh on which the products were deposited. The inset depicts an STEM image of a single chain, revealing the corresponding distribution of $\mathrm{Fe}$ and $\mathrm{C}$ elements in the chain. Note that the carbon signal from the iron carbide was difficult to measure compared with that of the iron, and the presence of the double carbon layer on the copper grid decreased the accuracy in determining this distribution.

A high-resolution TEM (HRTEM) image (Fig. 3c) was taken of one nanosphere as shown in the inset of Fig. 3b, and the interplanar distance was measured at $0.37 \mathrm{~nm}$; this result was in agreement with that for the (011) facet of the $\mathrm{Fe}_{3} \mathrm{C}$ iron carbide. The corresponding selected area electron diffraction (SAED) pattern (Fig. $3 \mathrm{~d}$ ) confirmed that the nanosphere was a single crystal. Based on careful analysis, the nanosphere was identified as orthorhombic iron carbide, which is indexed to the (011), $(0 \overline{1} 1)$, and (002) facets. Moreover, the HRTEM indicated that the nanosphere was covered with a layer of amorphous carbon, which may have reduced its level of oxidation. Another clear HRTEM image of the nanosphere is provided in Fig. $3 \mathrm{e}$ in which an interplanar distance of $0.34 \mathrm{~nm}$ was measured in accordance with the $(01 \overline{1})$ facet of Hägg iron carbide, $\mathrm{Fe}_{5} \mathrm{C}_{2}$. The corresponding SAED

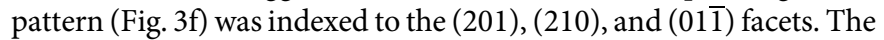
TEM results therefore confirmed that the micro-fibres were composed of submicron single-crystal iron carbide spheres $\left(\mathrm{Fe}_{3} \mathrm{C}\right.$ and $\mathrm{Fe}_{5} \mathrm{C}_{2}$ ).

$\mathrm{X}$-ray photoelectron spectroscopy (XPS) is a highly surface-sensitive technique for identifying the chemical state of materials; the XPS data are provided for the products in Fig. $4 a-b$. Because the iron carbide sphere surfaces were covered with amorphous carbon, we employed 3-kV Ar ion bombardment to remove the surface layer to a depth of $50 \mathrm{~nm}$. For the Fe 2p region, the two peaks at 707.45 and $720.28 \mathrm{eV}$ suggested the presence of iron carbide components ${ }^{20,21}$. The C1s spectrum was fitted with three components. The peak centred at $283.57 \mathrm{eV}$ was coincident with that of the $\mathrm{Fe}_{3} \mathrm{C}$ combination $^{22}$, while the other two peaks centred at 284.65 and $285.4 \mathrm{eV}$ corresponded to the $\mathrm{sp}^{2}$ - and $\mathrm{sp}^{3}$-bonded carbon ${ }^{23}$. Curve fitting of the $\mathrm{C}$ 1s spectra was performed using a standard least-squares algorithm to yield an $80 \%$ Gaussian Lorentzian-Gaussian peak shape. The measured full widths at half maximum (FWHM) for the $\mathrm{Fe}_{3} \mathrm{C}$, $\mathrm{sp}^{2}$, and $\mathrm{sp}^{3}$ were $0.98,0.88$, and $1.5 \mathrm{eV}$, respectively. The integrated areas of these peaks correspond to $\mathrm{Fe}_{3} \mathrm{C}, \mathrm{sp}^{2}$, and $\mathrm{sp}^{3}$ contents of $49.37,23.28$, and $27.35 \%$, respectively. A Shirley background was used in each fit, and the XPS data confirmed that the nanospheres consisted of iron carbide with far fewer Fe-O combinations resulting from the oxidation of iron carbide under $\mathrm{LAL}^{19}$.

A well-defined absorption band was observed at $\sim 300-400 \mathrm{~nm}$ in the UV-vis spectra of the products depicted in Fig. 4c and was assigned to the $\mathrm{Fe}_{3} \mathrm{C}$ iron carbide ${ }^{24}$. An absorption edge was also observed at wavelengths below $300 \mathrm{~nm}$ and was likely due to amorphous carbon ${ }^{25}$. These results indicate that the as-assembled $1 \mathrm{D}$ microfibres were formed from submicron single-crystalline iron carbide spheres.

Magnetisation characterisations of the 1D microfibres. The field dependence of the magnetisation of the products was measured at room temperature $(300 \mathrm{~K})$ and at low temperature $(5 \mathrm{~K})$; the results are provided in Fig. 5, which reveals ferromagnetic behaviour in the products. The $\mathrm{M}-\mathrm{H}$ plot in Fig. 5a indicates that the saturation magnetisation $\left(\mathrm{M}_{\mathrm{s}}\right)$ of the products was unexpectedly high, reaching 261 and $295 \mathrm{emu} / \mathrm{g}$ at 300 and $5 \mathrm{~K}$, respectively. The remnant magnetisation $\left(\mathrm{M}_{\mathrm{r}}\right)$ was measured at 16 and $25 \mathrm{emu} / \mathrm{g}$ at 300 and $5 \mathrm{~K}$, respectively, and the corresponding coercivity $\left(\mathrm{H}_{\mathrm{c}}\right)$ was measured at 140 and $277 \mathrm{Oe}$ at 300 and $5 \mathrm{~K}$, respectively, as indicated Fig. 5b. Surprisingly, the $\mathrm{M}_{\mathrm{s}}$ value of the products was 

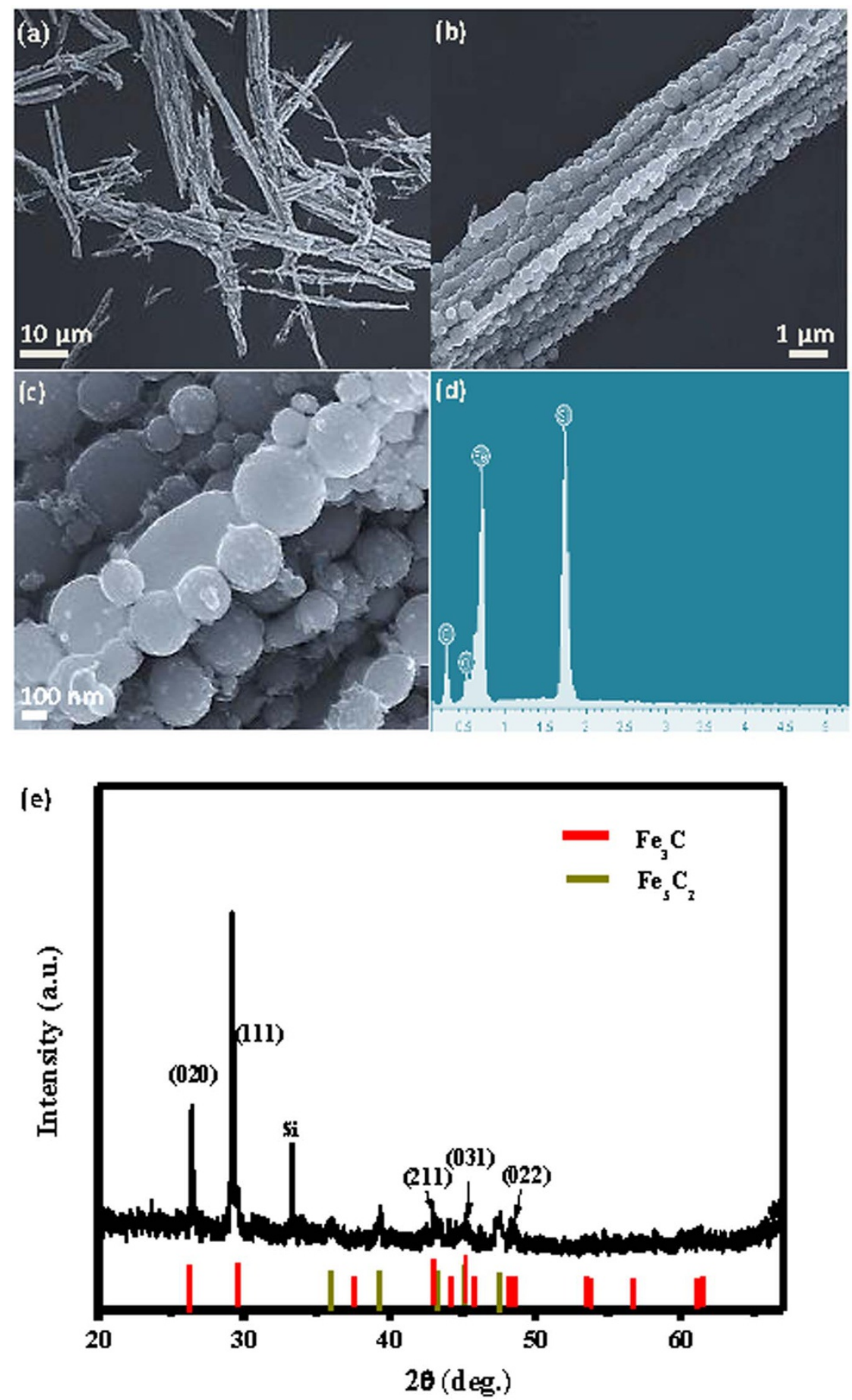

Figure $2 \mid$ SEM images of the micro-fibers fabricated at $9 \mathrm{~T}, 100 \mathrm{~mJ}$ and $5 \mathrm{~Hz}(\mathrm{a}-\mathrm{c})$, and corresponding EDS (d) and XRD patterns (e) for the products.

not only the best reported to date for iron nitride and carbide nanostructures ${ }^{18}$ but was also 5.5 -times greater than the $47 \mathrm{emu} / \mathrm{g}$ for $\mathrm{Fe}_{3} \mathrm{C}$ nanoparticles ${ }^{18}$ and greater than the $212 \mathrm{emu} / \mathrm{g}$ for bulk $\mathrm{Fe}^{26}$. The magnetic behaviour of ordered MNP nanostructures is known to depend strongly on the interparticle interactions ${ }^{27,28}$. Generally, the spin disorder on the surface and the surface oxidation of the MNPs reduce the total magnetic moment ${ }^{29-31}$. However, in our case, the thin amorphous carbon layer on the surface of the nanospheres prevented the MNPs from oxidising. Similar reports have demonstrated $M_{s}$ values above the bulk value ${ }^{32,33}$, which can be attributed to the special geometry of the magnetic nanostructures such as monopods and micro-octahedrons. Therefore, in our case, the abnormally giant saturation magnetisation of the MNP bundles was attributed to their unique structure and shape, whereas the exchange coupling between the nanospheres in the chains and that between the nanochains played crucial roles in enhancing the magnetic properties. The magnetic separability of the microfibres was tested in ethanol by placing a magnet near the glass bottle (see inset in Fig. 5a).

\section{Discussion}

We now discuss the formation of the MNP microfibre under MFLAL. The detailed mechanism for the nucleation and growth of NPs under LAL has been described previously ${ }^{18,34}$. When a strong 


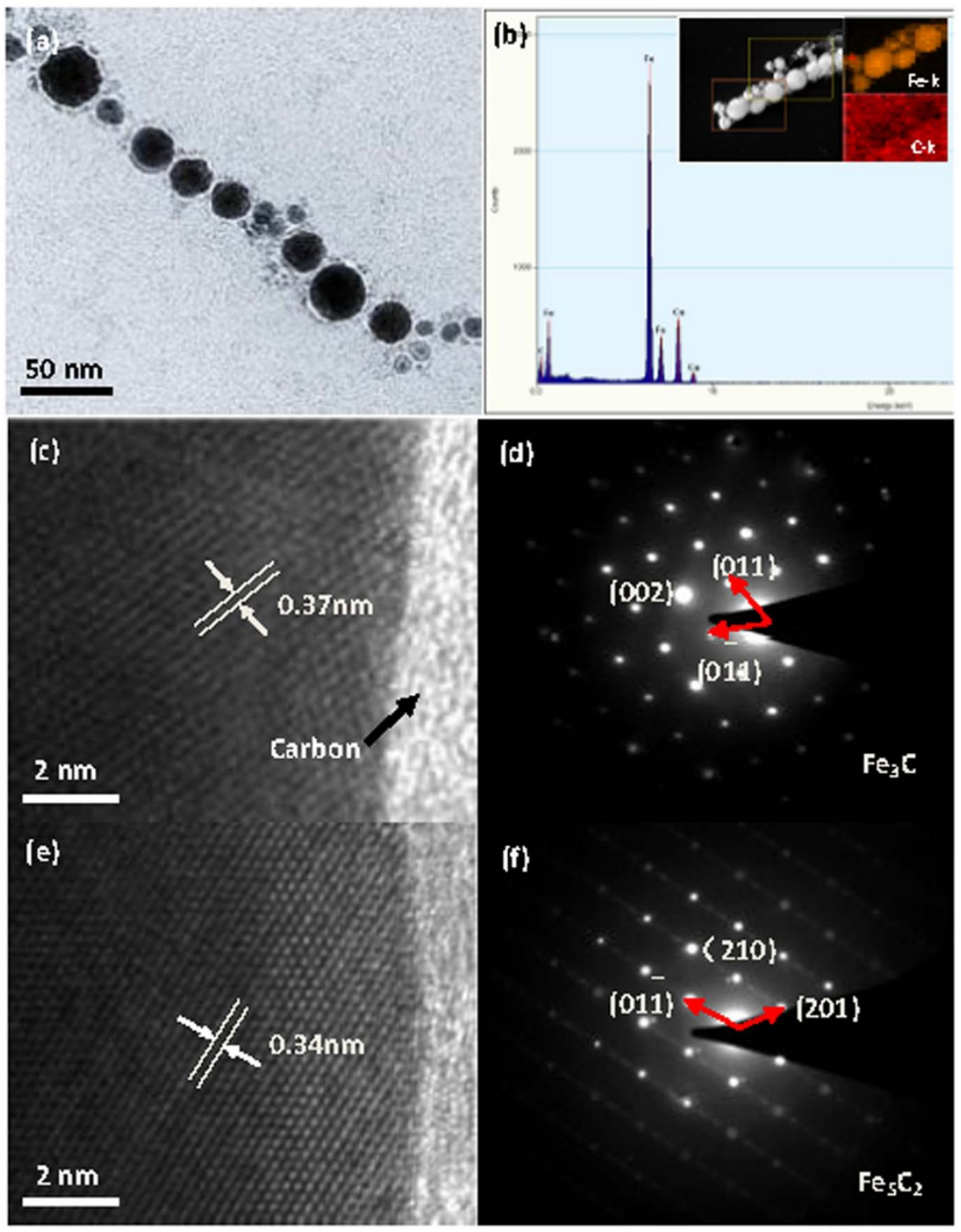

Figure 3 TEM bright-field image (a) of a single nanochain and the corresponding EDS (b), with inset STEM images. HRTEM images of two particles (c and $\mathrm{d}$ ), and their corresponding SAED patterns (e and f).

magnetic field is applied during LAL, the process becomes more complicated due to the motion of the charged particles induced by the Lorentz force. Previous reports have demonstrated changes in the plume structure and dynamics with enhanced emission, ionisation, and velocity in the presence of a magnetic field ${ }^{35-38}$. Thus, we hypothesise that the magnetic field affects the nanoparticle formation. Based on the aforementioned results, a strong magnetic field plays a critical role during the formation of the 1D MNP chains. The MNPs dispersed in a solution typically experience two attractive forces: the van der Waals (vdW) force $\mathrm{F}_{\mathrm{vdW}}$ and dipolar interactions $\mathrm{F}_{\mathrm{dd}}{ }^{38,39}$. The vdW force originates from the electromagnetic fluctuations produced by the constant movement of positive and negative charges within all atoms, molecules, and bulk materials ${ }^{40}$, whereas the dipolar interactions originate from two magnetic particles. When an external magnetic field is applied, the dipole-field force $\mathrm{F}_{\mathrm{m}}$ originating from $\nabla(\mathbf{m} \cdot \mathbf{H})$ should be considered because it can alter the energy distribution of the system ${ }^{40}$.

We calculated the three aforementioned interaction energies by considering a single linear chain system under a 9-T applied magnetic field (Fig. 6a). The idea was based on two basic assumptions: the MNPs were of the same size and were ordered one-by-one in a line along the magnetic field direction, and the nanospheres were magnetised in a spatially homogeneous fashion with a magnetic moment $\mathbf{m}$. Thus, we can consider three interaction energies: the vdW potential $U_{v d W}$, the magnetic dipole-dipole energy $\left(U_{d d}\right)$, and the magnetostatic energy $\mathrm{U}_{\mathrm{m}}$.

The isotropous vdW interaction was estimated using the Hamaker integral approximation according to the following formula for two spheres of radius $a$ separated by a centre-to-centre distance $\mathrm{r}^{39}$ :

$$
U_{v d W}=\frac{A}{3}\left[\frac{a^{2}}{r^{2}-(2 a)^{2}}+\frac{a^{2}}{r^{2}}+\frac{1}{2} \ln \left(\frac{r^{2}-(2 a)^{2}}{r^{2}}\right)\right]
$$

in which $\mathrm{A}$ is the Hamaker coefficient for ferrite particles (with a value of $\left.10^{-19} \mathrm{~J}\right)^{38,41}$. The vdW interaction depended on the particle size and the distance between two particles, as indicated in Fig. 6b, and decreased sharply when r/a slightly exceeded 2 . To simplify our experimental data, we set $\mathrm{r} / \mathrm{a}=2.1$ in the following discussion. The characteristic $U_{v d w}$ for an arbitrary NP with $\mathrm{r} / \mathrm{a}=2.1$ is a constant equal to $4.93 \times 10^{-20} \mathrm{~J}$.

The $U_{d d}$ represents the work required to bring two arbitrary dipoles with moments $\mathbf{m}_{\mathbf{1}}$ and $\mathbf{m}_{\mathbf{2}}$ from infinity to a finite separation of $\widehat{\mathbf{r}}^{39}$ :

$$
U_{d d}=\frac{\mathbf{m}_{1} \cdot \mathbf{m}_{2}-3\left(\mathbf{m}_{1} \cdot \widehat{\mathbf{r}}\right)\left(\mathbf{m}_{2} \cdot \widehat{\mathbf{r}}\right)}{4 \pi \mu_{0} r^{3}}
$$



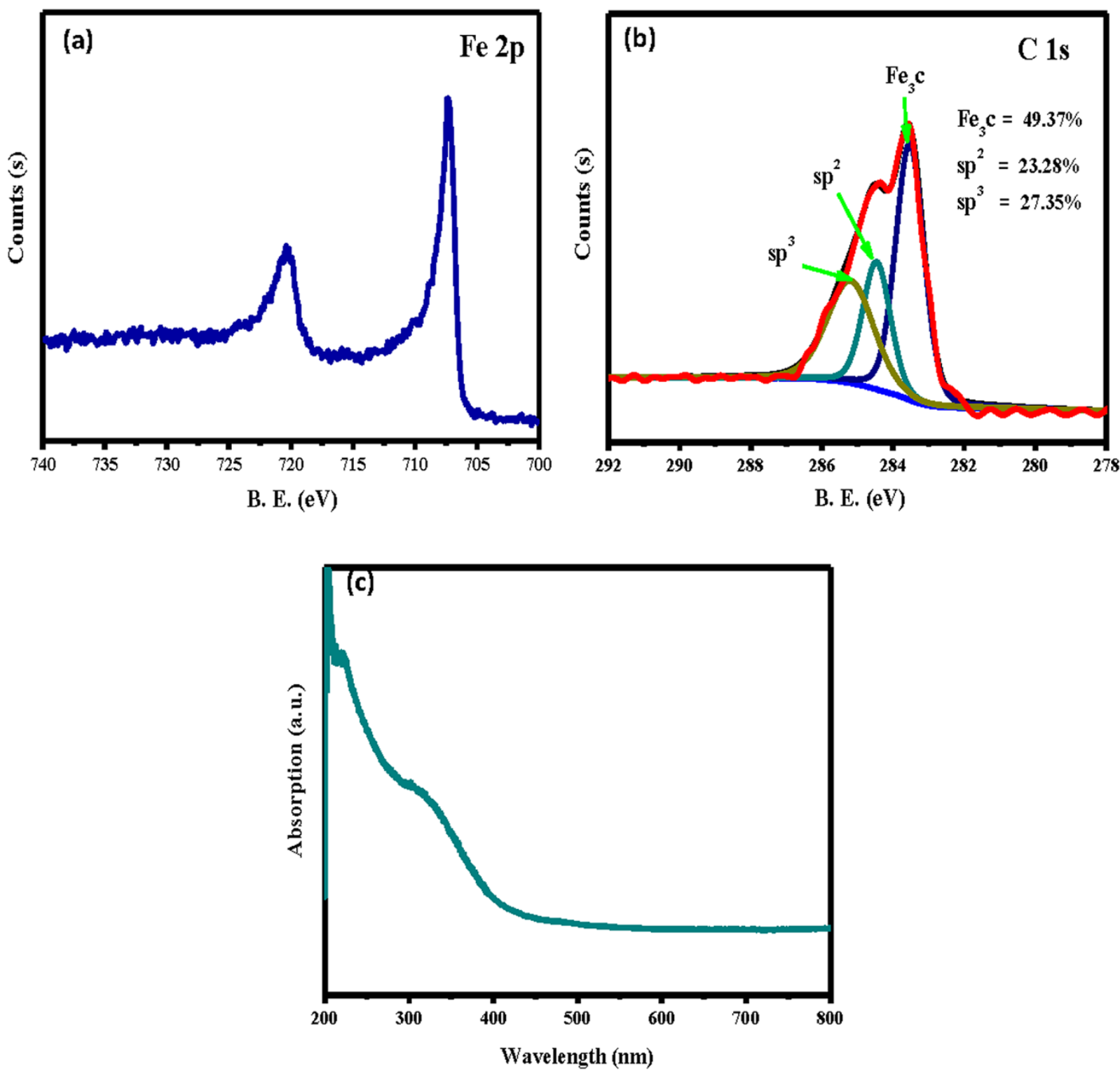

Figure $4 \mid$ XPS spectra (a-b) and UV-vis absorption spectrum (c) for the products.

for a magnetic sphere with a radius a, a particle volume $V=\frac{4}{3} \pi a^{3}$ and a magnetic moment $\mathbf{m}=\mu_{0} V \mathbf{M}$ in which $\mu_{0}$ is the permeability of vacuum and $\mathbf{M}$ is the saturation magnetisation equated with that of the bulk material, $\mathrm{M}_{\mathrm{s}}^{\text {bulk }}$. For two identical dipoles in a line, as in our case (Fig. 6a), $\mathbf{m}_{1} \cdot \mathbf{m}_{2}=m^{2}, \mathbf{m}_{1} \cdot \widehat{\mathbf{r}}=\mathbf{m}_{2} \cdot \widehat{\mathbf{r}}=m$, the interaction is attractive with a magnitude of $-m^{2} / 2 \pi \mu_{0} r^{3}$ or $-\frac{8}{9} \frac{\pi \mu_{0} a^{6} M^{2}}{r^{3}}$. For iron carbide, the $\mathrm{M}_{\mathrm{s}}^{\text {bulk }}$ value is $140 \mathrm{emu} / \mathrm{g}^{18}$ or $8.54 \times 10^{5} \mathrm{~A} / \mathrm{m}$, and the characteristic $U_{d d}$ at room temperature for a $100-\mathrm{nm} \mathrm{NP}$ is $-2.76 \times$ $10^{-16} \mathrm{~J}$.

The magnetostatic energy of a dipolar particle in an external field $\mathbf{H}$ is given by $U_{m}=-\mathbf{m} \cdot \mathbf{H}^{39}$. In our experiment, $\mathrm{H}=9 \mathrm{~T}$; thus, the $U_{m}$ of a $100-\mathrm{nm}$ MNP was estimated at $-3.58 \times 10^{-15} \mathrm{~J}$. For a $100-$ $\mathrm{nm} \mathrm{NP}, U_{m}$ is $\sim 10^{5}$ and $U_{d d}$ is $\sim 10^{4}$-times larger than $U_{\mathrm{vdw}}$. Note that these two interaction energies $U_{d d}$ and $U_{m}$ are strongest when $\mathbf{m}$ is the along the magnetic field direction, and they scale linearly with the particle volume such that the interactions between the particles are too weak to induce self-assembly. The $U_{d d}$ and $U_{m}$ values for 20-, 50-, 100-, 200-, 300-, and 500-nm particles are provided in Fig. $6 \mathrm{c}$ and have magnitudes of $\sim 10^{-18}-10^{-14}$ and $\sim 10^{-17}-10^{-13}$, respectively. For particles larger than $100 \mathrm{~nm}$, the magnetostatic energy dominates the vdW interactions $\left(\sim 10^{5}\right)$, resulting in a facile self-assembly of the $1 \mathrm{D}$ MNP chains. For particles smaller than $20 \mathrm{~nm}$, under a uniform 9-T magnetic field, the magnetostatic energy is 1000 -times greater than the vdW potential. Even if the magnetic field is removed, the $U_{d d}$ is still exceeds the $U_{v d W}$; therefore, the chain structure is easily preserved even when the magnetic field is removed. In our experiments, the nanospheres larger than $100 \mathrm{~nm}$ were obviously connected-or tended to align-along the magnetic lines of force, favouring the formation of linear chains. The $U_{d d}$ is anisotropic, changes with the relative position of the two dipoles and is written as $m^{2}\left(1-3 \cos ^{2} \alpha\right) / \mathrm{r}^{3}$ in which $\alpha$ ranges from $0^{\circ}$ to $90^{\circ}$. At the critical angle of $54.09^{\circ}, U_{d d}$ approaches zero. When $0 \leq \alpha<$ $54.09^{\circ}$, the interaction is attractive, but is repulsive for $54.09^{\circ}<\alpha \leq$ $90^{\circ}$. This dipole-dipole interaction maintains the force balance for the nanospheres, thus allowing the formation of the microfibres depicted in Fig. 6a. In our case, the average particle size ranged from 200-300 nm, which is larger than that typically reported for $\mathrm{LAL}^{19}$. We hypothesised that the laser-induced melting mechanism proposed by Koshizaki plays an important role in fabricating a magnetic nanochain via MF-LAL ${ }^{42-44}$. Throughout the laser ablation process, 

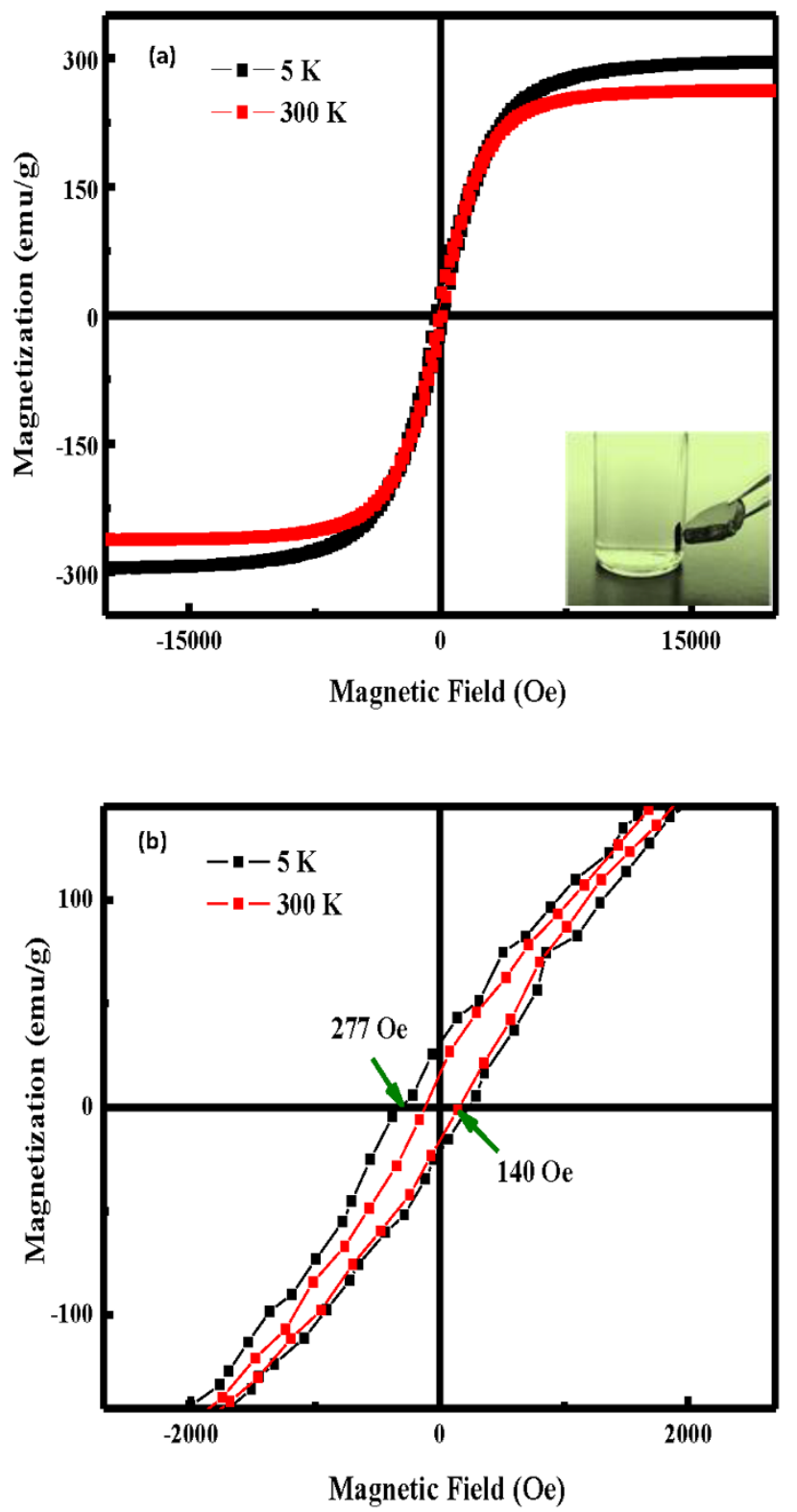

Figure 5 | Magnetic properties of the products; the magnetic migration of the products toward a magnet occurred within a few seconds (see inset in a).

the laser irradiation induced melting of the agglomerated NPs into submicron spheres. In our case, the fluence values used in the MFLAL were similar to those used in the laser irradiation of colloids ${ }^{42-44}$.

The previously described calculations provide a general understanding of the MNP microfibre formation. First, the strong magnetic field magnetises the nanospheres produced via LAL. However, the magnetostatic energy is at least one order of magnitude greater than the thermal energy $\left(\mathrm{k}_{\mathrm{B}} \mathrm{T}\right.$ in which $\mathrm{k}_{\mathrm{B}}$ is Boltzmann's constant and $\mathrm{T}$ is the absolute temperature; e.g., for $300 \mathrm{~K}, \mathrm{k}_{\mathrm{B}} \mathrm{T}$ is $4.14 \times$ $\left.10^{-21} \mathrm{~J}\right)^{34}$ and can overcome the thermal fluctuations to guide the particle movement. The nanospheres can then attract each other and tend to form linear chains along the line of magnetic force due to the head-to-tail alignment of the dipoles. Because the dipolar interaction and magnetostatic energies are considerably greater than the repulsive vdW potential, the $1 \mathrm{D}$ nanochains can be assembled.

The given the calculations indicate that the applied magnetic field plays a crucial role in assembling the MNP chain. To confirm this role, a control experiment was conducted in which the magnetic field
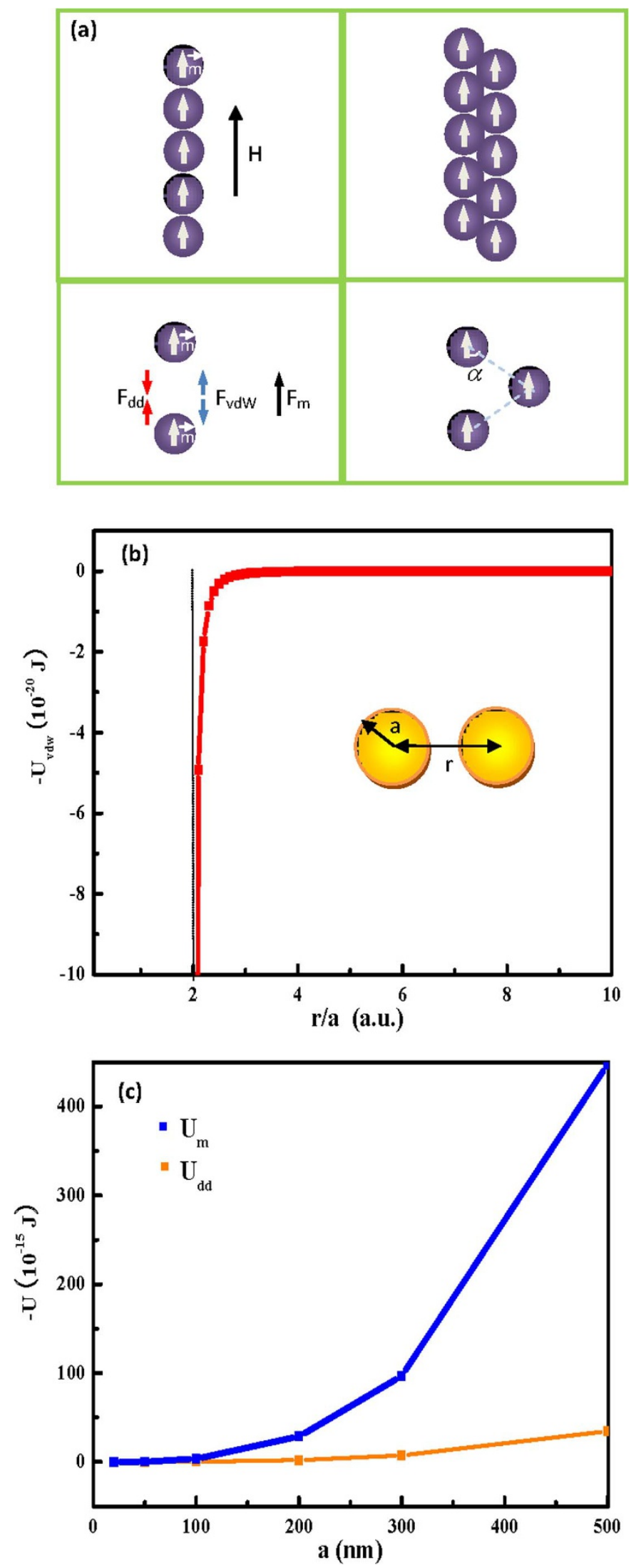

Figure $6 \mid$ A physical model illustration (a) of a 1D chain, and an analysis of the forces between two nanospheres under the applied field. A plot of the $\mathrm{vdW}$ potential against $\mathrm{r} / \mathrm{a}(\mathrm{b})$ and the dipolar-to-dipolar energy $\mathrm{U}_{\mathrm{dd}}$, and the magnetostatic energy of particles with different sizes (c). 

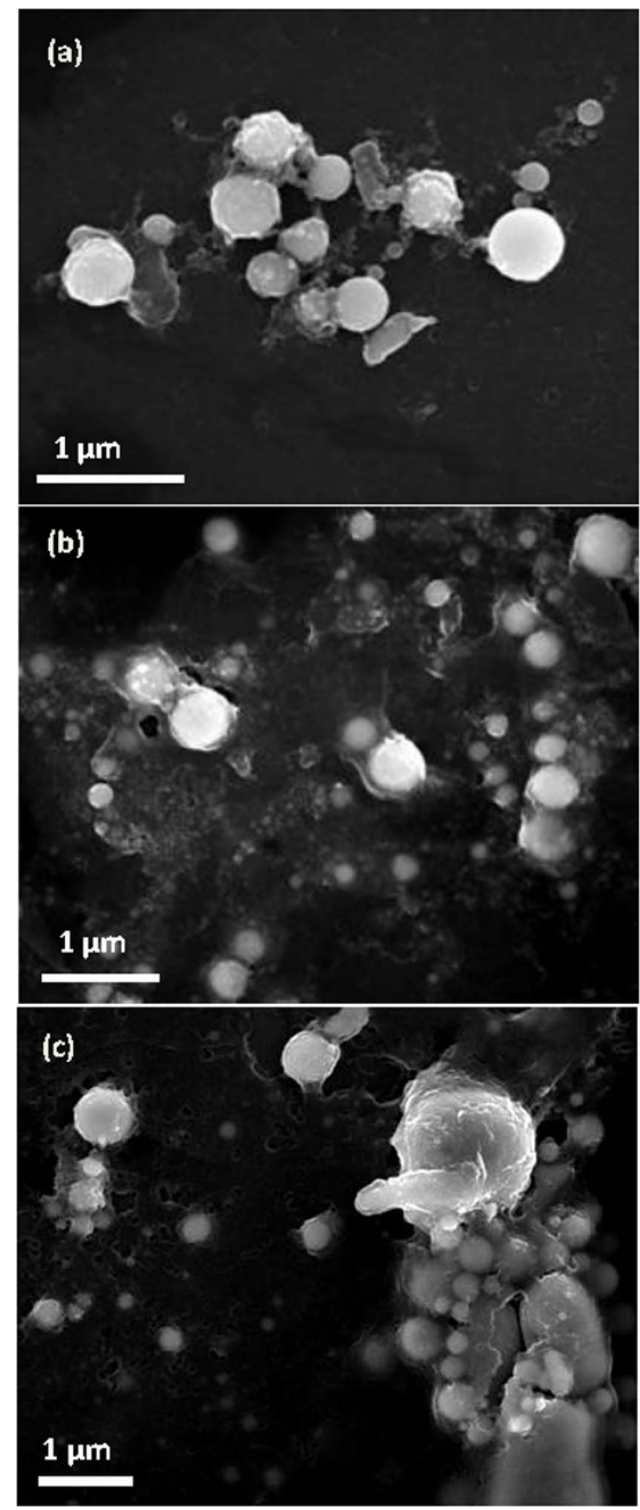

Figure 7 SEM images of laser ablation iron target in ethanol without the magnetic field. (a) the as-synthesized nanoparticles. The same sample placed in the magnetic field of $9 \mathrm{~T}$ after ablation at different time intervals: 2 hours (b) and 20 hours (c), respectively.

is present only after the synthesis and at various intervals. The submicron particles synthesised via LAL without any magnetic field but with all other conditions held constant are depicted in Fig. 7a. These nanoparticles are clearly disordered with no observable chains. Furthermore, the samples were firstly kept at room temperature for 2 and 20 hours, respectively, and then placed under magnetic field of $9 \mathrm{~T}$. The corresponding SEM images of the samples provided in Fig. $7 \mathrm{~b}$ and $\mathrm{c}$ display dispersed and random particles with no chains. Thus, we hypothesise that the magnetic field must be in situ during the laser ablation process to assemble the magnetic chains. In fact, our investigations indicated that the effects of the applied magnetic field on the chain fabrication via LAL and on the laser irradiation of colloids differ because LAL actually consists of two processes: laser ablation followed by the laser irradiation of the colloids. Therefore, our results demonstrated that ordered 1D chain bundles can be fabricated when a magnetic field is applied in situ during LAL. However, some disordered short chains form when the magnetic field is applied during the laser irradiation of the colloids.
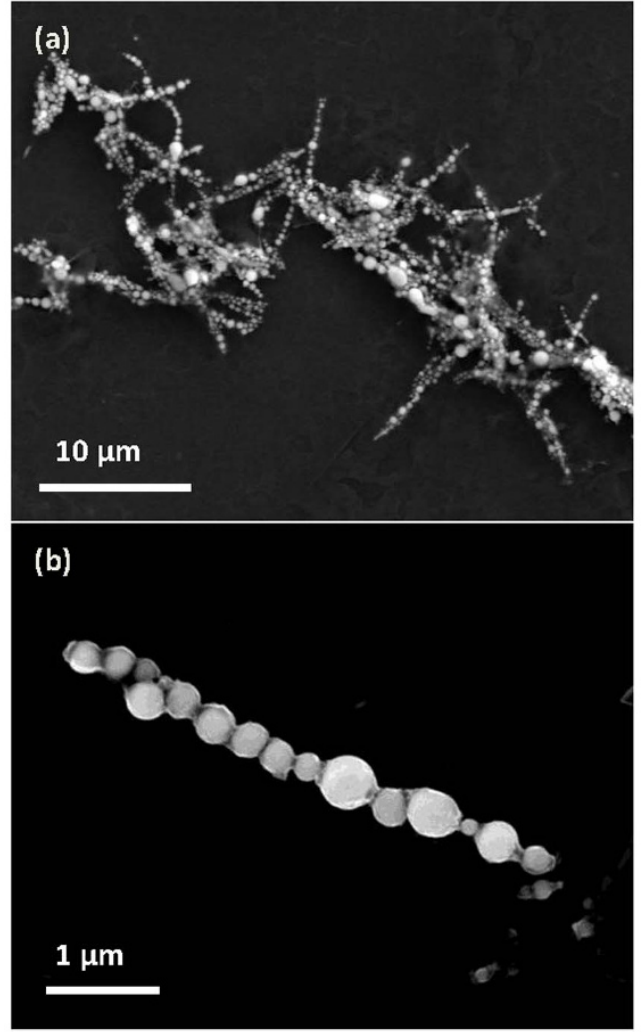

Figure 8 SEM images of the cobalt-based magnetic chains (a) fabricated by MF-LAL, and (b) an individual chain.

Amendola et al. reported the laser ablation of an iron target in different organic liquids for the synthesis of nanoparticles including iron carbide ${ }^{24}$. However, they did not characterise any magnetic properties of the as-synthesised nanoparticles. Additionally, Jakobi et al. reported the synthesis of magnetic Sm-Co and Ni-Fe alloys using LAL and the subsequent fabrication of short and disordered chains via polymerisation of the a nanoparticle/epoxy resin composite synthesised in an external magnetic field ${ }^{45}$. This two-step process involved the synthesis of magnetic nanoparticles via LAL without a magnetic field followed by the fabrication of short and disordered chains via polymerisation in an external magnetic field. Accordingly, we have developed a new one-step method involving magnetic fieldassisted laser ablation in liquid (MF-LAL) to assemble 1D iron carbide microfibres with giant magnetisation. This novel technique allows researchers to choose and design interesting solid targets for fabricating MNP microfibres. For example, we used cobalt as a solid target and ethanol as a liquid to assemble cobalt-based magnetic chains via MF-LAL as shown in Fig. 8, which implies that MF-LAL could become a universal technique for the assembly of magnetic chains and chain-like structures. In fact, the fabrication of $1 \mathrm{D}$ chains during MF-LAL is a relatively complex process with physical mechanisms that vary with the conditions. For example, during laser ablation, nanoparticles sometimes form inside a cavitation bubble ${ }^{46}$. Therefore, in our case, some fabricated fibres may form inside the bubble or during its collapse.

Based on the previous discussions, the effects of the applied magnetic field on the fabrication of $1 \mathrm{D}$ chains via MF-LAL can be summarised as follows: In the first stage of laser ablation, the applied magnetic field not only induces the $1 \mathrm{D}$ chain formation of magnetic NPs but also forces these 1D chains to form a bundle. Then, the applied magnetic field can enhance the growth of the $1 \mathrm{D}$ chain bundles. A schematic illustration of the $1 \mathrm{D}$ chain fabrication via MF-LAL is provided in Fig. 9. 


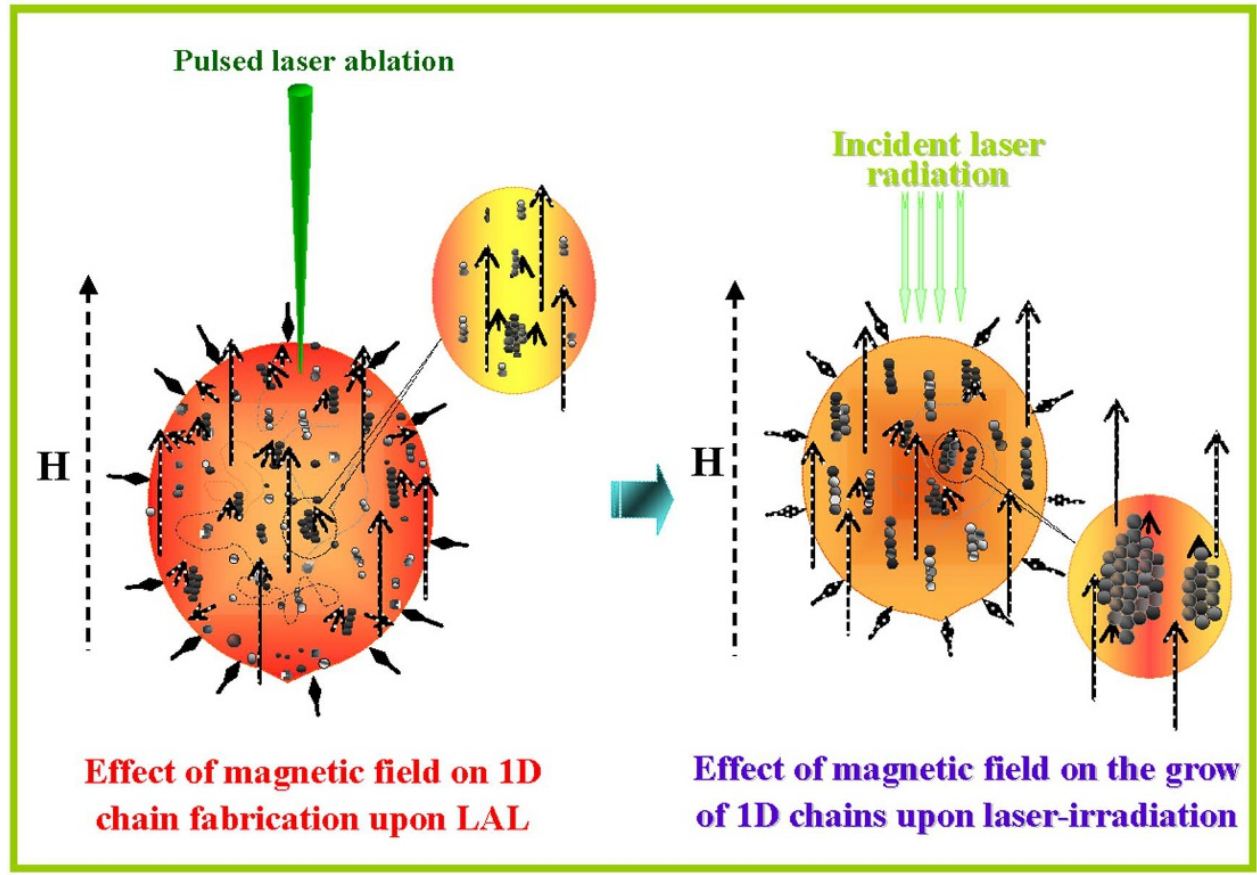

Figure 9 | Schematic illustration of 1D chain fabrication upon MF-LAL.

In summary, iron carbide microfibres were assembled via a simple, rapid, green, and relatively inexpensive route. These magnetic, ordered micro-fibres were constructed from 1D chains of submicron iron carbide spheres. Their saturation magnetisation was found to be $261 \mathrm{emu} / \mathrm{g}$ at room temperature, the best value reported to date for iron nitride and carbide nanoparticles and greater than that of bulk Fe. These findings make this unique magnetic nanostructure promising for a variety of biomedical and electronic applications.

\section{Methods}

Assembly of the magnetic microfibres. Laser ablation was performed using a Qswitch Nd:YAG laser device (Quanta-Ray Pro-250-, Newport Corporation, SpectraPhysics Div, Mountain View, CA 94043, U.S.A.) with a wavelength of $532 \mathrm{~nm}$, a pulse width of $10 \mathrm{~ns}$, a repeating frequency of $5 \mathrm{~Hz}$, and a pulse energy of $100 \mathrm{~mJ} /$ pulse (a schematic illustration of the magnetic field-assisted LAL is provided in the Supplementary Information). The solid iron target (99.99\% purity) used as the starting material was initially attached to the bottom of the glass chamber. Ethanol solvent ( $>99.7 \%$ pure) was slowly poured into the chamber until the target was submerged to a depth of $\sim 5-8 \mathrm{~mm}$. The container was then placed at the centre of the magnetic field, and the laser was focused onto the surface of the iron target with a focal length of $300 \mathrm{~mm}$. A steady, strong, and uniform 9-T magnetic field was then applied, and the laser ablation of the iron target in the strong magnetic field was performed. The interaction lasted $90 \mathrm{~min}$, and the solvent was collected for further measurements.

Characterising the structural and magnetic properties. Scanning electron microscopy (SEM) images were obtained using a JSM-7600F field emission scanning electron microscope operated at $15 \mathrm{kV}$ equipped with an EDS. XRD was performed using a D8 Advance X-ray diffractometer with $\mathrm{Cu} \mathrm{K} \alpha$ radiation $(\lambda=1.54056 \AA$, $40 \mathrm{kV}, 30 \mathrm{~mA}$ ), and transmission electron microscopy (TEM) was conducted using an FEI Tecnai G2 F30 instrument at an accelerating voltage of $300 \mathrm{kV}$. These techniques were used to identify the crystal structure and morphology of the products. The composition of the particle surface was analysed via X-ray photoelectron spectroscopy (XPS) (ESCA Lab250), and UV-vis spectroscopy was carried out with a UV 3150 spectrophotometer (Shimadzu, Japan). The magnetic properties of the samples were measured on a Quantum Design PPMS-9T SQUID magnetometer.

1. Varón, M. et al. Dipolar magnetism in ordered and disordered low-dimensional nanoparticle assemblies. Sci. Rep. 3, 1234; doi:10.1038/srep01234 (2013).

2. He, L., Wang, M. S., Ge, J. P. \& Yin, Y. D. Magnetic assembly route to colloidal responsive photonic nanostructures. Acc. Chem. Res. 45, 1431-1440 (2012).
3. Kinsella, J. M. \& Ivanisevic, A. Magnetotransport of one-dimensional chains of $\mathrm{CoFe}_{2} \mathrm{O}_{4}$ nanoparticles ordered along DNA. J. Phys. Chem. C 112, 3191-3193 (2008).

4. Goubault, C., Fermigier, J. P., Baudry, M., Bertrand, J. \& Bibette, E. Flexible magnetic filaments as micromechanical sensors. Phys. Rev. Lett. 91, 260802 (2003).

5. Zeng, H., Li, J., Liu, J. P., Wang, Z. L. \& Sun, S. H. Exchange-coupled nanocomposte magnets by nanoparticle self-assembly. Nature 420, 395-398 (2002).

6. Sudfeld, D. et al. New magnetic nanoparticles for biotechnology. IEEE Trans. Magn. 28, 2601 (2002).

7. Koenig, A. et al. Magnetic force probe for nanoscale biomolecules. Phys. Rev. Lett. 95, 128301 (2005).

8. Doyle, P. S., Bibette, J., Bancaud, A. \& Viovy, J. L. Self-assembled magnetic matrices for DNA separation chips. Science 295, 2237-2238 (2002).

9. Puntes, V. F., Gorostiza, P., Aruguete, D. M., Bastus, N. G. \& Alivisatos, A. P. Collective behavior in two-dimensional cobalt nanoparticle assemblies observed by magnetic force microscopy. Nat. Mater. 3, 263-268 (2004).

10. Chen, J. et al. Collective dipolar interactions in self-assembled magnetic binary nanocrystal superlattice membranes. Nano Lett. 10, 5103-5108 (2010).

11. Ku, J. Y., Aruguete, D. M., Alivisatos, A. P. \& Geissler, P. L. Self-assembly of magnetic nanoparticles in evaporating solution. J. Am. Chem. Soc. 133, 838-848 (2011).

12. Tang, Z. \& Kotov, N. A. One-dimensional assemblies of nanoparticles: preparation, properties, and promise. Adv. Mater. 17, 951-962 (2005).

13. Pileni, M. P. Nanocrystal self-assemblies: Fabrication and collective properties. J. Phys. Chem. B 105, 3358-3371 (2001).

14. Tang, Z., Kotov, N. A. \& Giersig, M. Spontaneous organization of single CdTe nanoparticles into luminescent nanowires. Science 297, 237-240 (2002).

15. Tang, Z., Ozturk, B., Wang, Y. \& Kotov, N. A. Simple preparation strategy and one-dimensional energy transfer in CdTe nanoparticle chains. J. Phys. Chem. B 108, 6927-3931 (2004).

16. Butter, K., Bomans, P. H. H., Frederik, P. M., Vroege, G. J. \& Philipse, A. P. Direct observation of dipolar chains in iron ferrofluids by cryogenic electron microscopy. Nat. Mater. 2, 88-91 (2003).

17. Wang, H. et al. Magnetic-field-induced formation of one-dimensional magnetite nanochains. Langmuir 25, 7135-7139 (2009).

18. Giordano, G., Kraupner, A., Wimbush, S. C. \& Antonietti, M. Iron Carbide: An Ancient Advanced Material. Small 17, 1859-1862 (2010).

19. Yang, G. W. Laser ablation in liquids: Applications in the synthesis of nanocrystals. Prog. Mater. Sci. 52, 648-698 (2007).

20. Zhou, J. S. et al. Oxidation conversion of carbon-encapsulated metal nanoparticles to hollow nanoparticles. Chem. Mater. 21, 3730-3737 (2009).

21. Yang, C., Zhao, H. B., Hou, Y. L. \& Ma, D. $\mathrm{Fe}_{5} \mathrm{C}_{2}$ nanoparticles: A facile bromideinduced synthesis and as an active phase for Fischer-Tropsch synthesis. J. Am. Chem. Soc. 134, 15814-15821 (2012). 
22. Goretzki, H., Rosenstiel, P. V. \& Mandziej, S. Small area MXPS- and TEMmeasurements on temper-embrittled $12 \% \mathrm{Cr}$ steel. Fres. Z. Anal. Chem. 333, 451-452 (1989).

23. Stuart, T., Jackson, A. \& Nuzzo, R. G. Determining hybridization differences for amorphous carbon from the XPS C1s envelope. Appl. Surf. Sci. 90, 195-203 (1995).

24. Amendola, V., Riello, P. \& Meneghetti, M. Magnetic nanoparticles of iron carbide, iron oxide, iron @ iron oxide, and metal iron synthesized by laser ablation in organic solvents. J. Phys. Chem. C 115, 5140-5146 (2011).

25. Amendola, V., Polizzi, S. \& Meneghetti, M. Free silver nanoparticles synthesized by laser ablation in organic solvents and their easy functionalization. Langmuir 23, 6766-6770 (2007).

26. Gao, C. \& Doyle, W. D. Quantitative correlation of phase structure with the magnetic moment in rf sputtered Fe-N films. J. Appl. Phys. 73, 6579-6581 (1993).

27. Han, D. H., Wang, J. P. \& Luo, H. L. Crystallite size effect on saturation magnetization of fine ferrimagnetic particles. J. Magn. Magn. Mater. 136, 176-182 (1994).

28. Jeong, U., Teng, X. W., Wang, Y., Yang, H. \& Xia, Y. N. Superparamagnetic colloids: controlled synthesis and niche applications. Adv. Mater. 19, 33-60 (2007).

29. Ye, J., Chen, Q. W., Qi, H. P. \& Tao, N. Formation of nickel dendritic crystals with peculiar orientations by magnetic-induced aggregation and limited diffusion. Cryst. Growth \& Des. 8, 2465-2468 (2008).

30. Giordano, C., Kraupner, A., Wimbush, S. C. \& Antonietti, M. Iron carbide: An ancient advanced material. Small 6, 1859-1862 (2010).

31. Meffre, A. et al. A simple chemical route toward monodisperse iron carbide nanoparticles displaying tunable magnetic and unprecedented hyperthermia properties. Nano Lett. 12, 4722-4728 (2012).

32. Gu, L. \& Shen, H. X. Facile one-pot synthesis of multi-armed $\mathrm{Fe}_{3} \mathrm{O}_{4}$ nanocrystals. J. Alloys and Compounds 472, 50-54 (2009).

33. Zhang, J. H. et al. Formation, characterization, and magnetic properties of $\mathrm{Fe}_{3} \mathrm{O}_{4}$ microoctahedrons. J. Crystal Growth 308, 159-165 (2007).

34. Ibrahimkutty, S., Wagener, P., Menzel, A., Plech, A. \& Barcikowski, S. Nanoparticle formation in a cavitation bubble after pulsed laser ablation in liquid studied with high time resolution small angle x-ray scattering. Appl. Phys. Lett 101, 103104 (2012).

35. Kokai, F., Koga, Y. \& Heimann, R. B. Magnetic field enhanced growth of carbon cluster ions in the laser ablation plume of graphite. Appl. Surf. Sci. 96, 261-266 (1996).

36. Harilal, S. S. et al. Confinement and dynamics of laser-produced plasma expanding across a transverse magnetic field. Phys. Rev. E 69, 026413 (2004).

37. Tachiki, Minoru. \& Takeshi, Kobayashi. Manipulation of laser ablation plume by magnetic field application. Jpn. J. appl. Phys. 38, 3642 (1999).

38. Lalatonne, Y., Richardi, J. \& Pileni, M. P. Van der Waals versus dipolar forces controlling mesoscopic organizations of magnetic nanocrystals. Nat. Mater. 3, 121-125 (2004).
39. Bishop, K. J. M., Wilmer, C. E., Soh, S. \& Grzybowski, B. A. Nanoscale forces and their uses in self assembly. Small 14, 1600-1630 (2009).

40. Ge, J. P., He, L., Hu, Y. X. \& Yin, Y. D. Magnetically induced colloidal assembly into field-responsive photonic structures. Nanoscale 3, 177-183 (2011).

41. Morimoto, H. \& Haekawa, T. Dynamic analysis of a ferromagnetic colloidal system. Int. J. Mod. Phys. B 13, 2085-2092 (2002).

42. Tsuji, T. et al. Preparation and investigation of the formation mechanism of submicron-sized spherical particles of gold using laser ablation and laser irradiation in liquids. Phys. Chem. Chem. Phys. 15, 3099-3107 (2013).

43. Li, X. et al. Fabrication of crystalline silicon spheres by selective laser heating in liquid medium. Langmuir 27, 5076-5080 (2011).

44. Wang, H. et al. Single-crystalline rutile $\mathrm{TiO} 2$ hollow spheres: room-temperature synthesis, tailored visible-light-extinction, and effective scattering layer for quantum dot-sensitized solar cells. J. Am. Chem. Soc. 133, 19102-19109 (2011)

45. Jakobi, J., Petersen, S., Menéndez-Manjón, A., Wagener, P. \& Barcikowski, S. Magnetic alloy nanoparticles from laser ablation in cyclopentanone and their embedding into a photoresist. Langmuir 26, 6892-6897 (2010).

46. Wagener, P., Ibrahimkutty, S., Menzel, A., Plech, A. \& Barcikowski, S. Dynamics of silver nanoparticle formation and agglomeration inside the cavitation bubble after pulsed laser ablation in liquid. Phys. Chem. Chem. Phys. 15, 3068-3074 (2013).

\section{Acknowledgements}

The National Basic Research Program of China (2014CB931700), NSFC (11004253), the Natural Science Foundation of Guangdong Province (S2011040001673), the Science Foundation of Guangdong Province under Grant No. sybzzxm201104 and State Key Laboratory of Optoelectronic Materials and Technologies supported this work.

\section{Author contributions}

G.W.Y. designed the experiments; Y.L. carried out the experiments and calculations and data analysis; P.L., J.X., H.B.L. assisted with some of the experiments; C.X.W. and G.W.Y guided the work and the analysis. Y.L. and G.W.Y. wrote the paper.

\section{Additional information}

Competing financial interests: The authors declare no competing financial interests.

How to cite this article: Liang, Y. et al. A microfibre assembly of an iron-carbon composite with giant magnetisation. Sci. Rep. 3, 3051; DOI:10.1038/srep03051 (2013). 